\title{
Dynamics of Discrete-Time Sliding-Mode-Control Uncertain Systems With a Disturbance Compensator
}

\author{
Shaocheng Qu, Member, IEEE, Xiaohua Xia, Fellow, IEEE, and Jiangfeng Zhang
}

\begin{abstract}
In this paper, dynamical behaviors of the discretetime sliding-mode-control (DSMC) uncertain systems are studied. First, a discrete reaching law with a disturbance compensator is presented, and a sliding-mode controller is designed by using the proposed reaching law. Second, the time steps for the system trajectories to converge to the switching manifold are found. Then, a quasi-sliding-mode domain (QSMD) of the DSMC uncertain systems is obtained, and the system dynamics of the DSMC systems in QSMD are described. Finally, numerical simulations are given to demonstrate the effectiveness of the proposed strategies.
\end{abstract}

Index Terms-Discrete-time uncertain systems, disturbance compensator (DSMC), dynamics, reaching law, sliding-mode control (SMC).

\section{INTRODUCTION}

A $\mathrm{S}$ a general design approach for robust control systems, sliding-mode-control (SMC) strategies have received wide attention, and the relevant results can be found in various publications [1]-[4], [22], [29]-[31]. The need for research in discrete-time SMC (DSMC) systems is evident. A primary reason is that most controllers nowadays are implemented in discrete time. Usually, there are two types of DSMC systems: one associated with the SMC of discrete-time systems [5]-[7], [9]-[15] and the other resulting from discretization of the SMC of continuous-time systems [16]-[25].

DSMC systems cannot be directly transformed from their continuous counterparts by means of simple equivalence [9]. Recently, some intrinsic dynamic properties within the discretized SMC systems have been found, such as periodic orbits

The work of S. Qu was supported by the National Natural Science Foundation of China under Grant 61074046/F030107, and by the Central China Normal University (CCNU) through the colleges basic research and operation of the Ministry of Education under the Self-Determined International Cooperation Project CCNU13F025.

S. Qu is with the Department of Electronics and Information Engineering, College of Physical Science and Technology, Central China Normal University, Wuhan 430079, China (e-mail: qushaocheng@ mail.ccnu.edu.cn).

X. Xia is with the Centre of New Energy Systems, Department of Electrical, Electronics and Computer Engineering, University of Pretoria, Pretoria 0002, South Africa (e-mail: xxia@ postino.up.ac.za).

J. Zhang is with the Centre of New Energy Systems, Department of Electrical, Electronics and Computer Engineering, University of Pretoria, Pretoria 0002, South Africa, and also with the Department of Electronic and Electrical Engineering, University of Strathclyde, Glasgow G1 1XW, U.K. (e-mail: zhang@up.ac.za).
[16]-[21] and delta modulation effects in the sliding direction [18]. The SMC of discrete-time systems is important, particularly when the implementation of the controller is realized by using computers with relatively low sampling frequencies [5][7], [9]-[15].

Some of the work on the DSMC systems focuses on the reaching conditions of the DSMC systems. There are normally two types of reaching conditions: the inequality conditions [5][7] and the equality conditions [8]-[12], [14]. Gao and Hung [8] propose a reaching law which is based on the equality type of the reaching conditions; then, an SMC controller is designed by using the reaching law. Gao et al. [9] further develop a discrete reaching law, which can steer the switching function to converge to the switching manifold in finite time and, then, to undergo a zigzag motion in the vicinity of the switching manifold. A notion of the quasi-sliding mode (QSM) in the vicinity of the switching manifold is developed in [9].

Usually, the system trajectories of the DSMC systems cannot reach the origin but only tend to reach a chattering surrounding the origin [9], [10]. There are some methods developed to alleviate the chattering. A reaching law is developed to overcome the chattering in [11]. A strategy combining the reaching law and the fast-output-sampling method with output feedback is developed in [12]. A two-scale reaching law with Euler velocity estimation is proposed in [14]. A no-switching type of DSMC is discussed, and an $o\left(T^{2}\right)$ boundary layer in the vicinity of the switching manifold is found in [15]; however, such a layer is inevitable for any sampled-data systems by nature of the zero-order-holder $(\mathrm{ZOH})$ sampling method. By redefining a multivalued sign function, a chattering-free digital SMC with state observer and disturbance rejection is presented in [23][25]. There have been other efforts to reduce the chattering, such as state observers, disturbance compensators, and adaptive techniques [13], [14].

The objectives of this paper are to develop a discrete reaching law with a disturbance compensator and to describe the dynamics of the DSMC uncertain systems designed by using the proposed reaching law. First, a measure of the uncertain parameters and external disturbances is established by the deviations between the switching function and the reaching law, and a discrete reaching law with a disturbance compensator is presented. Second, the time steps for the system trajectories to converge to the switching manifold are found, and a QSM domain (QSMD) of the closed-loop SMC system is obtained. Third, the system dynamics of the closed-loop system in the vicinity of the switching manifold are described. Finally, simulation examples are given to verify the theoretical results. 


\section{SYSTEM DESCRIPTION}

Consider a general description of an uncertain single-input system

$$
\dot{x}=A x+B u+D f
$$

where $x \in R^{n}$ is the state, $u \in R^{1}$ is the control, the generalized uncertainty $f \in R^{l}$ includes the variations of system parameters, control uncertainties, and external disturbances, and $A, B$, and $D$ are constant matrices of appropriate dimensions. The following assumptions are needed throughout this paper.

Assumption I: The pair $(A, B)$ is completely controllable.

Assumption II: The generalized uncertainty is smooth and bounded.

Assumption III: The generalized uncertainty satisfies the matching condition [1], i.e.,

$$
\operatorname{rank}[B, D]=\operatorname{rank}[B] .
$$

To alleviate chattering, a discrete-time approach which takes the sample-hold effects into account is necessary. By applying control through a ZOH sampling, i.e., $u(t)=u(k)$ for time $t \in$ $[k T,(k+1) T]$, where $T$ is the sampling period, the discretetime representation of the system (1) with the $\mathrm{ZOH}$ is written as

$$
x_{k+1}=\Phi x_{k}+\Gamma u_{k}+d_{k}
$$

where $x_{k}=x(k T), \quad u_{k}=u(k T), \quad d_{k}=d(k T), \quad \Phi=e^{A T}$, $\Gamma=\int_{0}^{T} e^{A \tau} d \tau B$, and $d_{k}=\int_{0}^{T} e^{A \tau} D f((k+1) T-\tau) d \tau$.

It should be pointed out that $d_{k}$ in (3) is obtained under the assumption that $D f$ in (1) is considered constant over the sampling period, i.e., the matched condition after discretization only holds in the sense of approximation.

The switching function is defined as

$$
s_{k}=C x_{k}
$$

where the vector $C \in R^{1 \times n}$ is to be designed such that the system will achieve the desired dynamics when traveling along the switching manifold

$$
S=\left\{x_{k} \mid C x_{k}=0\right\} .
$$

Similar to the case of continuous-time systems, once system (3) reaches the switching manifold (5) and is maintained on it, the order of the closed-loop system (3) will be reduced to $(n-1)$. The desired dynamics of the sliding mode, governed by this $(n-1)$ order system, can be designed by an appropriate choice of vector $C$. Furthermore, $C \Gamma \neq 0$ is assumed.

The control objective is to steer the system trajectories to converge toward the switching manifold $S$ defined in (5) and also to travel to the origin along the switching manifold $S$.

Now, let us specify how the QSM and the reaching condition are understood in this paper.

Definition 1: System (3) is said to be in a QSM in the $\Delta$ vicinity of the switching manifold (5) if a motion of the system (3) satisfies $\left\{x_{k}|| C x_{k} \mid \leq \Delta\right\}$ for all $k \geq k^{*}$ ( $k^{*}$ is a constant integer). The specified space domain where the QSM occurs is called the QSMD, and the positive constant $\Delta$ is called the width of the QSMD.

Definition 2: System (3) is said to satisfy the reaching condition of the QSM in the $\Delta$ vicinity of the switching manifold (5) if the following conditions hold: When $s_{k}>\Delta$, then $-\Delta \leq$ $s_{k+1}<s_{k}$; when $s_{k}<-\Delta$, then $s_{k}<s_{k+1} \leq \Delta$; and when $\left|s_{k}\right| \leq \Delta$, then $\left|s_{k+1}\right| \leq \Delta$.

The following result is needed in the proof of Lemma 2 .

Lemma 1 [18], [28]: For a 1-D delta-modulated feedback system in the form of a discrete-time dynamics, $x^{+}=f(x) \triangleq$ $a x-\Delta \operatorname{sgn}(a x)$, where $x^{+}$denotes the system state at the next discrete-time step, $a$ is a real number, and $\operatorname{sgn}(x)$ is defined as

$$
\operatorname{sgn}(x)= \begin{cases}1, & \text { if } x \geq 0 \\ -1, & \text { if } x<0\end{cases}
$$

The following results are valid.

1) If $|a|=1$, then $\Omega=[-\Delta, \Delta]$ is a global attractor on $(-\infty, \infty)$.

2) If $|a|<1$, then the global attractor $\Omega$ is a set of two points: $\{-\Delta /(1+|a|), \Delta /(1+|a|)\}$.

3) If $0 \leq a<1$, then two points $\{-\Delta /(1+|a|), \Delta /(1+$ $|a|)\}$ are two-periodic points; if $-1<a<0$, then two points $\{-\Delta /(1+|a|), \Delta /(1+|a|)\}$ are (one-periodic) fixed points.

\section{Design of the DSMC Systems With the PROPOSED REACHING LAW}

The design of the DSMC systems includes the following two steps.

1) Determine a sliding-mode controller $u_{k}$ and a switching function (4) such that the closed-loop system (3) satisfies the reaching condition in Definition 2. It shows that the closed-loop system will be forced to the QSMD defined in Definition 1. Furthermore, once entering into the QSMD, the system trajectories of the closed-loop system will stay within QSMD and never escape it.

2) Further design a switching function (4) such that the closed-loop system (3) restricted to the switching manifold (5) is asymptotically stable.

Gao et al. developed a discrete reaching law in [9]. On the one hand, a sliding-mode controller can be synthesized by this reaching law. On the other hand, it not only can guarantee that the closed-loop system satisfies the reaching condition but also can be directly used to dictate the dynamics of the switching function of the closed-loop SMC system.

\section{A. New Reaching Law With a Disturbance Compensator}

The following new reaching law with a disturbance compensator is proposed:

$$
\begin{aligned}
s_{k+1}=(1-q T) s_{k}-\varepsilon T \operatorname{sgn}\left(s_{k}\right)+C d_{k} \\
\quad-\sum_{i=2}^{k}\left\{s_{i}-\left[(1-q T) s_{i-1}-\varepsilon T \operatorname{sgn}\left(s_{i-1}\right)\right]\right\}
\end{aligned}
$$


where $T$ is the sampling period, $\varepsilon>0$ is a switching parameter, $q>0$ is a converging parameter, and $0<1-q T<1$ is required. It is assumed that the change rate of the generalized uncertainty $d_{k}$ is bounded as follows:

$$
\begin{aligned}
\delta_{k} & \triangleq C\left(d_{k}-d_{k-1}\right) \\
\left|\delta_{k}\right| & \leq \delta \leq \varepsilon T, \quad k=0,1,2, \ldots .
\end{aligned}
$$

It implies that the uncertainties are assumed to be slowly time varying.

It follows from (6) that

$$
\begin{aligned}
& s_{k}=(1-q T) s_{k-1}-\varepsilon T \operatorname{sgn}\left(s_{k-1}\right)+C d_{k-1} \\
&-\sum_{i=2}^{k-1}\left\{s_{i}-\left[(1-q T) s_{i-1}-\varepsilon T \operatorname{sgn}\left(s_{i-1}\right)\right]\right\} \\
& \sum_{i=2}^{k}\left\{s_{i}-\left[(1-q T) s_{i-1}-\varepsilon T \operatorname{sgn}\left(s_{i-1}\right)\right]\right. \\
&=s_{k}-\left[(1-q T) s_{k-1}-\varepsilon T \operatorname{sgn}\left(s_{k-1}\right)\right] \\
& \quad+\sum_{i=2}^{k-1}\left\{s_{i}-\left[(1-q T) s_{i-1}-\varepsilon T \operatorname{sgn}\left(s_{i-1}\right)\right]\right\} .
\end{aligned}
$$

Substituting (9) into (10) yields

$$
\sum_{i=2}^{k}\left\{s_{i}-\left[(1-q T) s_{i-1}-\varepsilon T \operatorname{sgn}\left(s_{i-1}\right)\right]=C d_{k-1} .\right.
$$

Equation (11) shows that the accumulated deviations between the switching function $s_{i}$ and $(1-q T) s_{i}-\varepsilon T \operatorname{sgn}\left(s_{i}\right)$ reveal the generalized uncertainty at the $k-1$ steps. Su et al. [15] and Morgan and Özgüner [26] predict and replace $d_{k-1}$ by $x_{k}-\Phi x_{k-1}-\Gamma u_{k-1}$ in (11), and it is successfully implemented for robotics manipulators in [27].

Substituting (11) into (6), we obtain an explicit expression of the proposed reaching law (6)

$$
s_{k+1}=(1-q T) s_{k}-\varepsilon T \operatorname{sgn}\left(s_{k}\right)+C\left(d_{k}-d_{k-1}\right)
$$

which will be discussed later.

\section{B. Design of a Sliding-Mode Controller}

In the following, a sliding-mode controller for DSMC uncertain systems is designed by using the proposed reaching law (6).

Substituting the system dynamics (3) into the switching function (4) yields

$$
\begin{aligned}
s_{k+1} & =C x_{k+1} \\
& =C \Phi x_{k}+C \Gamma u_{k}+C d_{k} .
\end{aligned}
$$

Comparing (13) to the improved reaching law (6) gives

$$
\begin{aligned}
s_{k+1}= & (1-q T) s_{k}-\varepsilon T \operatorname{sgn}\left(s_{k}\right)+C d_{k} \\
& -\sum_{i=2}^{k}\left[s_{i}-(1-q T) s_{i-1}+\varepsilon T \operatorname{sgn}\left(s_{i-1}\right)\right] \\
= & C \Phi x_{k}+C \Gamma u_{k}+C d_{k} .
\end{aligned}
$$

Note that $C \Gamma \neq 0$; the controller $u_{k}$ can be solved from the previous equation

$$
\begin{aligned}
u_{k}=-(C \Gamma)^{-1}\{ & C \Phi x_{k}-(1-q T) s_{k}+\varepsilon T \operatorname{sgn}\left(s_{k}\right) \\
& \left.+\sum_{i=2}^{k}\left[s_{i}-(1-q T) s_{i-1}+\varepsilon T \operatorname{sgn}\left(s_{i-1}\right)\right]\right\}
\end{aligned}
$$

which is the sliding-mode controller designed by using the proposed reaching law (6). Note that the controller (14) does not depend on the uncertainties and therefore can be directly implemented.

Under the assumption

$$
\left|C\left(d_{k}-d_{k-1}\right)\right|=0
$$

the proposed reaching law (6) is simplified to the following reaching law [9, eq. (11)]:

$$
s_{k+1}=(1-q T) s_{k}-\varepsilon T \operatorname{sgn}\left(s_{k}\right) .
$$

Moreover, the sliding-mode controller (14) is reduced to the following sliding-mode controller [9, eq. (20)]:

$$
u_{k}=-(C \Gamma)^{-1}\left[C \Phi x_{k}-(1-q T) s_{k}+\varepsilon T \operatorname{sgn}\left(s_{k}\right)\right] .
$$

Remark 1: Comparing (6) and (16), it is noted that only iterative terms are added in the proposed reaching law (6). Furthermore, the iterative terms only consist of the historical information. It shows that all attributes of the reaching law in [9] are preserved. Compared to (17), only accumulated deviations are added in controller (14). This is very convenient when the proposed controller (14) is implemented by microprocessors.

Remark 2: Some other novel techniques on the reaching law can be incorporated into the proposed reaching law (6). For example, the two-scale reaching law in [14] can be applied to the proposed reaching law (6) by adding an adjusting parameter to $\varepsilon T$ in (6) (see [14, eq. (22)]). Nevertheless, it should be pointed out that the discrete dynamics (12) and (16) cannot reach the origin in view of the traditional definition of the sgn function in Lemma 1.

\section{System Dynamics of The DSMC System With THE PROPOSED REACHING LAW}

The DSMC systems designed by using the reaching law are not asymptotically stable, i.e., the system trajectories do not converge to zero asymptotically due to the switching with a finite frequency and system uncertainties. It will be shown hereinafter that, under certain conditions, system (3) with the switching manifold (5) and controller (14) is stable and the system trajectories are bounded.

If the generalized uncertainty $d_{k}$ satisfies (15), i.e., $d_{k}$ is zero or constant, the following Lemma 2 can be obtained.

Lemma 2: Consider the system (3) with the assumption (15); if the sliding-mode controller (14) is implemented, then the 
system trajectories of the closed-loop system (3) from any initial state will converge to the QSMD defined by

$$
s_{\Delta 1}=\left\{x_{k}|| C x_{k} \mid \leq \frac{\varepsilon T}{2-q T}\right\}
$$

and the following two points at the two ends of the QSMD

$$
\left\{\frac{-\varepsilon T}{2-q T}, \frac{\varepsilon T}{2-q T}\right\}
$$

are a global two-periodic attractor.

Proof: If the controller (14) is implemented for the system (3) with assumption (15), the dynamics of the switching function (4) of the closed-loop system (3) will be completely described by the reaching law (16). According to the aforementioned Lemma 1(b) and Lemma 1(c), and noting the assumption $0<1-q T<1$, the proof is straightforward.

Remark 3: If the sampling period $T$ is sufficiently small, then the QSMD (18) will be very close to the switching manifold (5). Moreover, the system trajectories in steady state will approach the origin. It should be indicated that the same conclusion (18) is found with a different method by Bartoszewicz in [10].

In the following, we divide the discussions on the dynamics of the DSMC uncertain system into two parts: One is how many steps that the system trajectories need to converge to the QSMD, and the other is system dynamics in the vicinity of the switching manifold.

\section{A. Convergence of System Trajectories Toward QSMD}

Theorem 1: Consider system (3) with assumptions (7) and (8); if the controller (14) is implemented, then the trajectories of the switching function (4) from any initial state will first cross the switching manifold (5) within $k^{*}+1$ steps at most, where $k^{*}=\left\lfloor m_{1 \mathrm{st}}^{*}\right\rfloor$

$$
m_{1 \mathrm{st}}^{*}=\log _{(1-q T)}\left(\frac{\varepsilon T-\delta}{\varepsilon T-\delta+q T\left|s_{0}\right|}\right) .
$$

$s_{0}$ is an initial point of the switching function, and $\left\lfloor m_{1 \mathrm{st}}^{*}\right\rfloor$ denotes the floor operation, i.e., the maximal integer bounded below the real number $m_{1 \mathrm{st}}^{*}$.

Proof: When the controller (14) is implemented for the system (3) with assumptions (7) and (8), the dynamics of the switching function of the closed-loop system will be described by (12). From (12) and (7), it yields that

$$
s_{k+1}=(1-q T) s_{k}-\varepsilon T \operatorname{sgn}\left(s_{k}\right)+\delta_{k} .
$$

In the following, we prove the theorem by contradiction according to (21).

Assume that there is an initial state, resulting in the initial point $s_{0}$ of the switching function (4), such that $s_{0}, s_{1}$, $\ldots, s_{k^{*}+1}$ do not change signs. There are two cases: $s_{0} \geq 0$ and $s_{0}<0$.

Case I: Assume that $s_{0} \geq 0$, and the system trajectories from the initial state do not cross the switching manifold in $k^{*}+1$ number of steps, i.e., $s_{m}$ are nonnegative for all $m \leq k^{*}+1$. It follows from (21) and (8) that

$$
\begin{aligned}
s_{1}= & (1-q T) s_{0}-\left(\varepsilon T-\delta_{0}\right) \\
s_{2}= & (1-q T) s_{1}-\varepsilon T+\delta_{1} \\
= & (1-q T)^{2} s_{0}-(1-q T)\left(\varepsilon T-\delta_{0}\right)-\left(\varepsilon T-\delta_{1}\right) \\
& \vdots \\
s_{m}= & (1-q T) s_{m-1}-\varepsilon T+\delta_{m-1} \\
= & (1-q T)^{m} s_{0}-(1-q T)^{m-1}\left(\varepsilon T-\delta_{0}\right)-\cdots \\
& -(1-q T)\left(\varepsilon T-\delta_{m-2}\right)-(1-q T)^{0}\left(\varepsilon T-\delta_{m-1}\right) \\
= & (1-q T)^{m} s_{0}-\sum_{i=0}^{m-1}(1-q T)^{m-1-i}\left(\varepsilon T-\delta_{i}\right) \\
\leq & (1-q T)^{m} s_{0}-\sum_{i=0}^{m-1}(1-q T)^{m-1-i}(\varepsilon T-\delta) \\
= & (1-q T)^{m}\left|s_{0}\right|-(\varepsilon T-\delta) \frac{1-(1-q T)^{m}}{q T} .
\end{aligned}
$$

Noting assumption (8) and that $0<1-q T<1$, it is straightforward to verify that the real number $m_{1 \mathrm{st}}^{*}$ in (20) satisfies

$$
(1-q T)^{m_{1 \mathrm{st}}^{*}}\left|s_{0}\right|-(\varepsilon T-\delta) c \frac{1-(1-q T)^{m_{1 \mathrm{st}}^{*}}}{q T}=0 .
$$

It follows from $k^{*}=\left\lfloor m_{1 \mathrm{st}}^{*}\right\rfloor$ that

$$
\begin{aligned}
s_{k^{*}+1} & \leq(1-q T)^{k^{*}+1}\left|s_{0}\right|-(\varepsilon T-\delta) \frac{1-(1-q T)^{k^{*}+1}}{q T} \\
& <(1-q T)^{m_{1 \mathrm{st}}^{*}}\left|s_{0}\right|-(\varepsilon T-\delta) \frac{1-(1-q T)^{m_{1 \mathrm{st}}^{*}}}{q T} \\
& =0
\end{aligned}
$$

which contradicts the assumption that $s_{m} \geq 0$ is nonnegative for all $m \leq k^{*}+1$.

Case II: Assume that $s_{0}<0$, and $s_{m}$ is negative for all $m \leq$ $k^{*}+1$; similarly, it follows that

$$
\begin{aligned}
s_{m} & =(1-q T)^{m} s_{0}+\sum_{i=0}^{m-1}(1-q T)^{m-1-i}\left(\varepsilon T+\delta_{i}\right) \\
& \geq(1-q T)^{m} s_{0}+\sum_{i=0}^{m-1}(1-q T)^{m-1-i}(\varepsilon T-\delta) \\
& =-\left[(1-q T)^{m}\left|s_{0}\right|-(\varepsilon T-\delta) \frac{1-(1-q T)^{m}}{q T}\right] .
\end{aligned}
$$

It can also be verified that the real number $m_{1 \mathrm{st}}^{*}$ in (20) satisfies

$$
(1-q T)^{m_{1 \mathrm{st}}^{*}}\left|s_{0}\right|-(\varepsilon T-\delta) \frac{1-(1-q T)^{m_{1 \mathrm{st}}^{*}}}{q T}=0 .
$$


It follows from $k^{*}=\left\lfloor m_{1 \mathrm{st}}^{*}\right\rfloor$ that

$$
\begin{aligned}
s_{k^{*}+1} & \geq-(1-q T)^{k^{*}+1}\left|s_{0}\right|+(\varepsilon T-\delta) \frac{1-(1-q T)^{k^{*}+1}}{q T} \\
& >-(1-q T)^{m_{1 \mathrm{st}}^{*}}\left|s_{0}\right|+(\varepsilon T-\delta) \frac{1-(1-q T)^{m_{1 \mathrm{st}}^{*}}}{q T} \\
& =0
\end{aligned}
$$

which is again a contradiction to the assumption that $s_{m}$ is all negative for all $m \leq k^{*}+1$.

The aforementioned two cases show that $k^{*}=\left\lfloor m_{1 \mathrm{st}}^{*}\right\rfloor$ is the smallest integer such that the system trajectories are guaranteed to first cross the switching manifold (5) within $k^{*}+1$ steps. The proof of Theorem 1 is completed.

If $\delta=0,(20)$ is reduced to

$$
m_{1 \mathrm{st}}^{* *}=\log _{(1-q T)}\left(\frac{\varepsilon}{\varepsilon+q\left|s_{0}\right|}\right)
$$

which shows that the system trajectories in Lemma 2 will first cross the switching manifold (5) in $\left\lfloor m_{1 \mathrm{st}}^{* *}\right\rfloor+1$ number of steps and eventually enter into the global two-periodic orbit (19) within the QSMD defined by (18).

\section{B. System Dynamics in the Vicinity of the Switching Manifold}

According to Theorem 1, the system trajectories from any initial state will cross the switching manifold in a finite number of steps. The following results characterize the system dynamics of the closed-loop system (3) in the vicinity of the switching manifold.

Theorem 2: The following results hold for the system (3) with assumptions (8) and controller (14).

1) The system trajectories from any initial state will enter into a QSMD defined by

$$
s_{\Delta 2}=\left\{x_{k}|| C x_{k} \mid \leq \varepsilon T+\delta\right\}
$$

i.e., the QSMD is globally attractive for the switching function of the closed-loop system.

2) Once the system trajectories enter into the QSMD, they cannot escape it.

3) Any system trajectories in QSMD will cross the switching manifold repeatedly within each $k^{* *}+1$ new steps, where $k^{* *}=\left\lfloor m_{2}^{*}\right\rfloor$ and

$$
m_{2}^{*}=\log _{(1-q T)}\left(\frac{\varepsilon T-\delta}{\varepsilon T-\delta+q T(\varepsilon T+\delta)}\right) .
$$

Proof: In a similar way as in the proof of Theorem 1, we can prove that the dynamics of the switching function of the closed-loop system (3) are described by (12).

1) If $s_{k}>0$, it follows from (8) that

$$
\begin{aligned}
s_{k+1} & =(1-q T) s_{k}-\varepsilon T+\delta_{k} \\
& <s_{k}-\varepsilon T+\delta_{k}<s_{k}
\end{aligned}
$$

namely, the sequence $\left\{s_{k}\right\}$ is decreasing as long as $s_{k}>0$. Similarly, it can be proved that the sequence $\left\{s_{k}\right\}$ is increasing as long as $s_{k}<0$. According to Theorem 1, the sequence $\left\{s_{k}\right\}$ will cross the switching manifold in a finite number of steps. Assuming that the sequence $\left\{s_{k}\right\}$ does not enter into the QSMD, then there are only two situations, i.e., there exists a positive integer $l$ such that Case I: $s_{0}>s_{1}>\ldots>s_{l}>0$, but $s_{l+1}<-(\varepsilon T+\delta)$ or

Case II: $s_{0}<s_{1}<\ldots<s_{l}<0$, but $s_{l+1}>(\varepsilon T+\delta)$.

In Case I, it follows that

$$
s_{l+1}=(1-q T) s_{l}-\varepsilon T+\delta_{l}
$$

but

$$
s_{l+1}<-\varepsilon T-\delta .
$$

This is impossible, since $s_{l}>0$ and $\left|\delta_{l}\right| \leq \delta \leq \varepsilon T$. Similarly, Case II is also impossible. It follows that the sequence $\left\{s_{k}\right\}$ will enter into the QSMD, i.e., the system trajectories from any initial state will enter into the QSMD defined by (23). The proof of Theorem 2(a) is completed.

2) By Theorem 2(a), the system trajectories from any initial state will enter into a QSMD. When $0 \leq s_{k} \leq \varepsilon T+\delta$, then

$$
s_{k+1}=(1-q T) s_{k}-\varepsilon T+\delta_{k} \geq-\varepsilon T-\delta
$$

also

$$
\begin{aligned}
s_{k+1} & =(1-q T) s_{k}-\varepsilon T+\delta_{k} \\
& \leq \varepsilon T+\delta-\varepsilon T+\delta_{k} \leq \varepsilon T+\delta .
\end{aligned}
$$

When $-(\varepsilon T+\delta) \leq s_{k}<0$, then

$$
s_{k+1}=(1-q T) s_{k}+\varepsilon T+\delta_{k} \leq \varepsilon T+\delta
$$

also

$$
\begin{aligned}
s_{k+1} & =(1-q T) s_{k}+\varepsilon T+\delta_{k} \\
& \geq-(\varepsilon T+\delta)+\varepsilon T+\delta_{k} \geq-(\varepsilon T+\delta) .
\end{aligned}
$$

It can be concluded from the aforementioned two cases that, once the system trajectories enter into the QSMD, they never escape it.

3) According to Theorem 2(a) and Theorem 2(b), the system trajectories will enter into the QSMD defined in (23) and never escape it. Assuming the worst case when $s_{0}= \pm(\varepsilon T+\delta)$ in (20), it is then straightforward to obtain the conclusion (24).

This completes the proof of Theorem 2.

In the following, we consider a special case: once the system trajectories have crossed the switching manifold, they will also cross the switching manifold in each successive step. This motion can adequately exhibit robust control of the DSMC systems [9], [10]. It is interesting to further characterize this behavior within the QSMD. 
The aforementioned description can be characterized as follows:

$$
\operatorname{sgn}\left(s_{k+2}\right)=-\operatorname{sgn}\left(s_{k+1}\right)=\operatorname{sgn}\left(s_{k}\right) .
$$

It follows from (12) that

$$
\begin{aligned}
s_{k+2}= & (1-q T) s_{k+1}-\varepsilon T \operatorname{sgn}\left(s_{k+1}\right)+C\left(d_{k+1}-d_{k}\right) \\
= & (1-q T)\left[(1-q T) s_{k}-\varepsilon T \operatorname{sgn}\left(s_{k}\right)+C\left(d_{k}-d_{k-1}\right)\right] \\
& -\varepsilon T \operatorname{sgn}\left(s_{k+1}\right)+C\left(d_{k+1}-d_{k}\right) \\
= & (1-q T)^{2} s_{k}+q T \varepsilon T \operatorname{sgn}\left(s_{k}\right) \\
& +(1-q T) C\left(d_{k}-d_{k-1}\right)+C\left(d_{k+1}-d_{k}\right) .
\end{aligned}
$$

It can be concluded from (26) that the $s_{k+2}$ and $s_{k}$ will have the same signs if the following condition holds:

$$
\left|(1-q T) C\left(d_{k}-d_{k-1}\right)+C\left(d_{k+1}-d_{k}\right)\right| \leq q T \varepsilon T .
$$

Solving (27) yields

$$
\delta \leq \frac{q T \varepsilon T}{2-q T} .
$$

Remark 4: Assume that the uncertainty boundary $\delta$ in (8) satisfies (28), once $s_{k}$ has crossed the switching manifold (5), then it also will cross the switching manifold (5) in all successive steps within the QSMD defined by (23). If the uncertainty boundary does not satisfy (28), it cannot maintain to cross the switching manifold in all successive steps, but the system trajectories still stay within QSMD defined by (23).

Finally, let us consider the system states of the closed-loop system.

Theorem 3: If the vector $C$ in (4) is chosen such that $\|M\|<$ 1 , where $M=\Phi-\Gamma(C \Gamma)^{-1} C \Phi$, and the uncertainty $d_{k}$ is bounded as $\left\|d_{k}\right\| \leq \zeta$, then the system states of the SMC system (3) with control (14) are bounded by

$$
\left\|x_{\infty}\right\| \leq \Psi(1-\|M\|)^{-1}
$$

where $\quad \Psi=\left\|\Gamma(C \Gamma)^{-1}\right\|[(1-q T)(\delta+\varepsilon T)+\varepsilon T]+\zeta(1+$ $\left.\left\|\Gamma(C \Gamma)^{-1} C\right\|\right)$.

Proof: Substituting control (14) into the system (3) yields

$$
x_{k+1}=M x_{k}+W_{k}+V_{k}
$$

where

$$
\begin{aligned}
W_{k} & =\Gamma(C \Gamma)^{-1}\left[(1-q T) s_{k}-\varepsilon T \operatorname{sgn}\left(s_{k}\right)\right] \\
V_{k} & =d_{k}-\Gamma(C \Gamma)^{-1} C d_{k-1} .
\end{aligned}
$$

Since $d_{k}$ is bounded by $\left\|d_{k}\right\| \leq \zeta$, then

$$
\left\|V_{k}\right\| \leq \zeta\left(1+\left\|\Gamma(C \Gamma)^{-1} C\right\|\right) .
$$

Furthermore, once the system trajectories have entered into the QSMD, then

$$
\begin{aligned}
\left\|W_{k}\right\| & \leq\left\|\Gamma(C \Gamma)^{-1}\right\|\left[(1-q T)\left\|s_{k}\right\|+\varepsilon T\right] \\
& \leq\left\|\Gamma(C \Gamma)^{-1}\right\|[(1-q T)(\delta+\varepsilon T)+\varepsilon T] .
\end{aligned}
$$

Noting definition $\Psi$, it follows from (30) that

$$
\begin{aligned}
\left\|x_{k+1}\right\| & =\left\|M x_{k}+W_{k}+V_{k}\right\| \\
& \leq\|M\|\left\|x_{k}\right\|+\left\|W_{k}\right\|+\left\|V_{k}\right\| \\
& \leq\|M\|\left\|x_{k}\right\|+\Psi
\end{aligned}
$$

iterating it for $n$ times from the $k$ th step, we have

$$
\left\|x_{k+n}\right\| \leq\|M\|^{n}\left\|x_{k}\right\|+\Psi \sum_{i=0}^{n-1}\|M\|^{n-1-i} .
$$

Therefore, if $\|M\|<1$, it follows from (31)-(33) that the state trajectories of the SMC system in steady state will be bounded by (29). This completes the proof of Theorem 3 .

\section{NUMERICAL EXAMPLES}

Consider the second-order system with time-varying uncertainties in the form of (1), where

$$
\begin{aligned}
A & =\left[\begin{array}{cc}
0 & 1 \\
5 & -2
\end{array}\right] \quad b=\left[\begin{array}{l}
0 \\
1
\end{array}\right] \\
D f & =\left[\begin{array}{c}
0 \\
a(1+2.2 \cos (0.5 \pi t))
\end{array}\right]
\end{aligned}
$$

and $a$ is an adjustable parameter. The initial state is assumed as $x(0)=\left[\begin{array}{ll}2.1 & 1\end{array}\right]^{\mathrm{T}}$.

The sampling period is chosen as $T=0.1$. Then, the discrete-time representation of the system with $\mathrm{ZOH}$ sampling is obtained as

$$
x_{k+1}=\Phi x_{k}+\Gamma u_{k}+d_{k}
$$

where

$$
\begin{aligned}
\Phi & =\left[\begin{array}{ll}
1.02351 & 0.09139 \\
0.45696 & 0.84073
\end{array}\right] \quad \Gamma=\left[\begin{array}{l}
0.00470 \\
0.09139
\end{array}\right] \\
d_{k} & =a \times\left[\begin{array}{ll}
0.10080 & 0.00470 \\
0.02351 & 0.09139
\end{array}\right]\left[\begin{array}{c}
0 \\
1+2.2 \cos (0.5 \pi K T)
\end{array}\right]
\end{aligned}
$$

and $C=\left[\begin{array}{ll}1 & 1\end{array}\right]$ in (4).

In the following, we discuss the system dynamics in three cases.

Case I: Consider the system (34) with an adjustable parameter $a=0$ (i.e., $d_{k}=0$ ); when the control (14) with parameters $q=5$ and $\varepsilon=3$ is implemented, then the switching function of the closed-loop SMC system is shown in Fig. 1. Here, the big switching parameter $\varepsilon=3$ is chosen so as to show the dynamics depicted by Lemma 2 . As stated by Lemma 2, the system trajectories starting from the initial state enter into the QSMD $s_{\Delta 1}=\left\{x_{k}|| C x_{k} \mid \leq 0.2\right\}$ as defined in (18), and the two points $\{-0.2,0.2\}$ at the two ends of the QSMD are a global two-periodic attractor. Moreover, it can be observed from Fig. 1 that the system trajectories first cross the switching manifold in $k^{* *}+1$ number of steps, where $k^{* *}=2$ is the maximal integer bounded below the real number 2.7370 . 


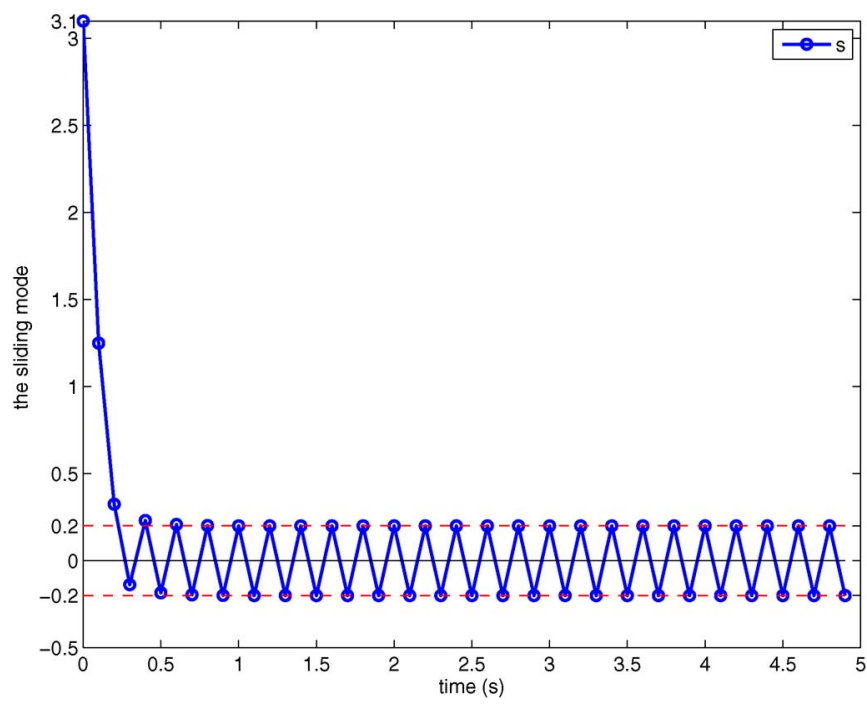

Fig. 1. Switching function with the reaching law in case I.

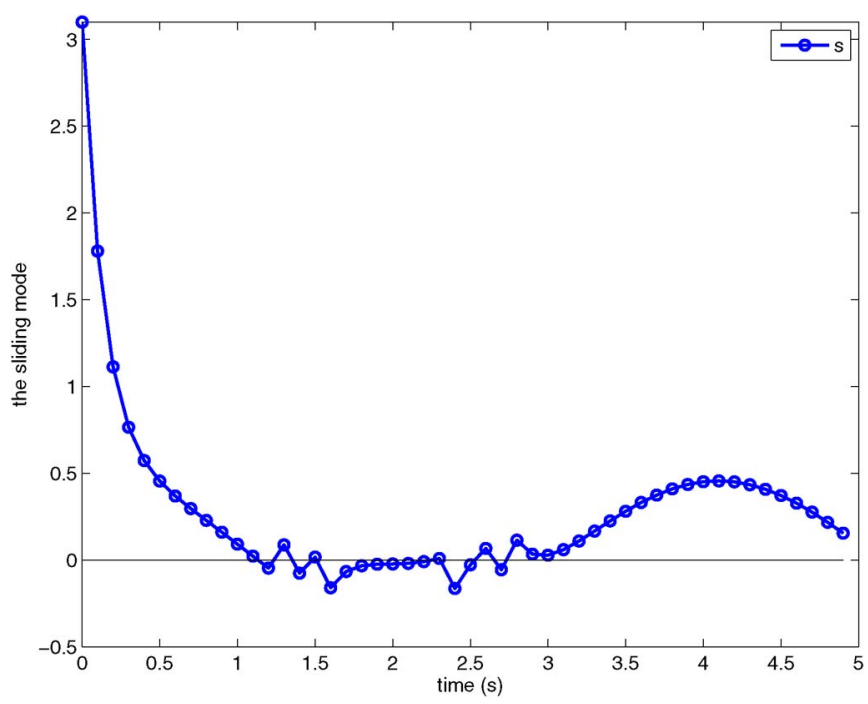

Fig. 2. Switching function with the reaching law in case II.

Case II: When the control (17) with parameters $q=5$ and $\varepsilon=1$ is implemented for the system (34) with an adjustable parameter $a=1$, then the switching function, system states, and controller are shown in Figs. 2-4, respectively. When the proposed SMC (14) with the same parameters is implemented, then the switching function, system states, and controller are shown in Figs. 5-7, respectively. By comparison, we can observe that the dynamics of the closed-loop system designed by using the proposed reaching law are effectively improved in the presence of uncertainties.

The uncertainty boundary $\delta=0.033070$ in (8) satisfies the condition (28), i.e., $\delta \leq(q T \varepsilon T / 2-q T)=$ 0.033333; Fig. 5 confirms Remark 4 that, once $s_{k}$ has crossed the switching manifold, it maintains to cross the switching manifold in all subsequent steps. The QSMD is defined as $s_{\Delta 2}=\left\{x_{k}|| C x_{k} \mid \leq \delta+\varepsilon T=0.13307\right\}$ by (23) in Theorem 2.

It should be indicated that the long sampling period $T=0.1$ is chosen in order to clearly exhibit the system

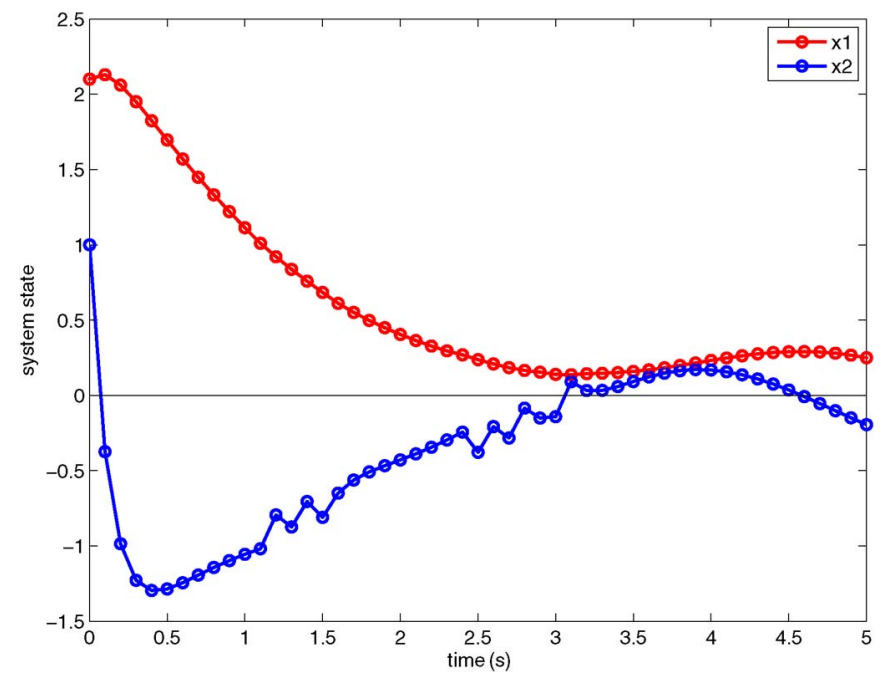

Fig. 3. System states with the reaching law in case II.

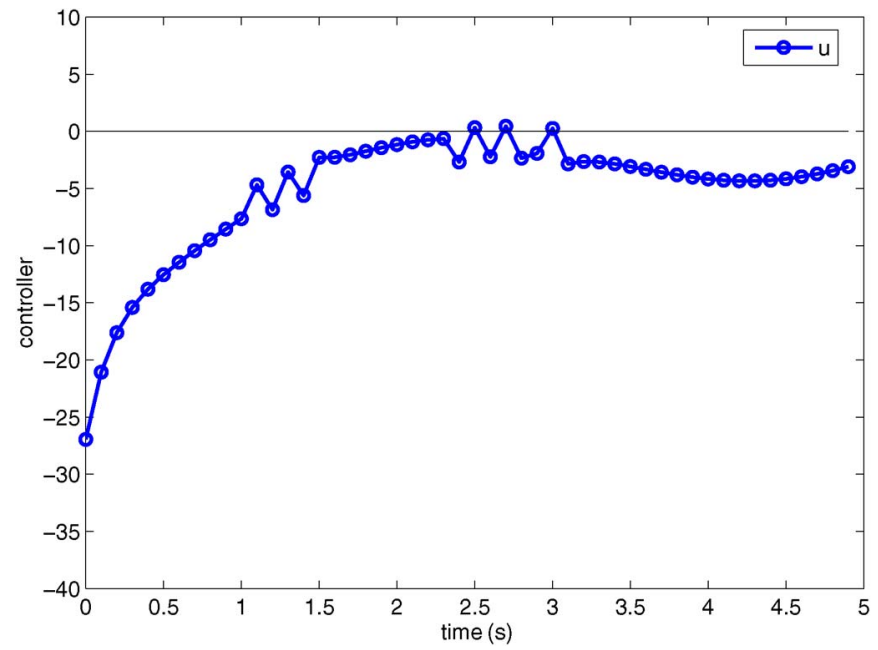

Fig. 4. Controller with the reaching law in case II.

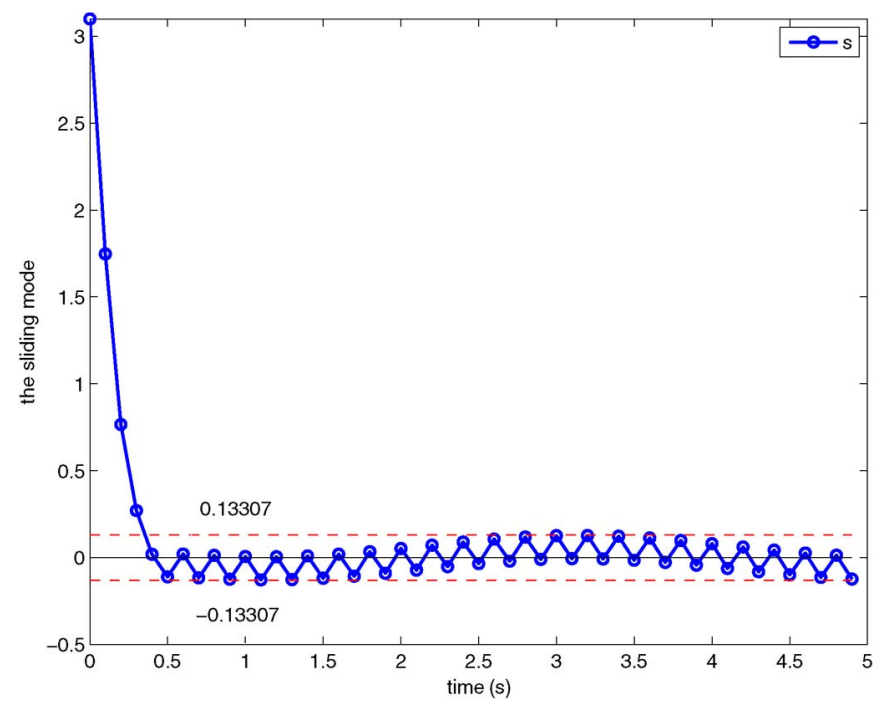

Fig. 5. Switching function with the improved reaching law in case II.

dynamics of the closed-loop system. If the sampling period $T=0.01$ and the same control parameters $q=5$ and $\varepsilon=1$ are implemented, then the magnitude of the controller will be alleviated as Fig. 8 . 


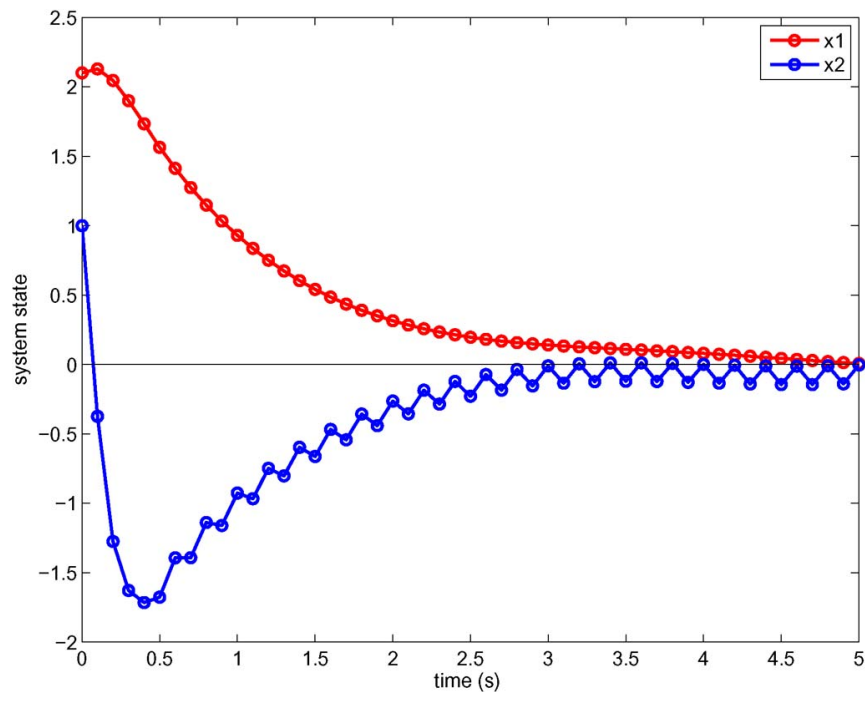

Fig. 6. System states with the improved reaching law in case II.

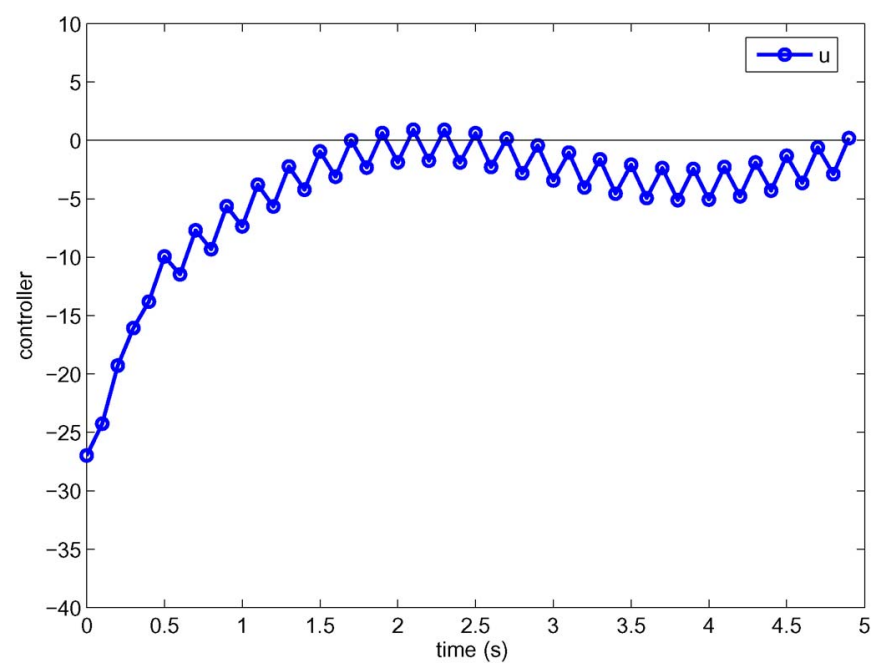

Fig. 7. Controller with the improved reaching law in case II.

Case III: Consider the system (34) with an adjustable parameter $a=1.05$; when the proposed SMC (14) with parameters $q=5$ and $\varepsilon=1$ is implemented, the switching function of the closed-loop system is shown in Fig. 9. Because the uncertainty boundary $\delta=0.034723$ in (8) and it does not satisfy the condition (28), i.e., $\delta>(q T \varepsilon T / 2-q T)=$ 0.033333 , it is observed from Fig. 9 that the sequence $\left\{s_{k}\right\}$ does not maintain to cross the switching manifold in all subsequent steps, but it still lies in the QSMD defined by $s_{\Delta 2}=\left\{x_{k}|| C x_{k} \mid \leq \delta+\varepsilon T=0.13472\right\}$ by (23) in Theorem 2; moreover, it is still attracting and invariant.

\section{CONCLUSION}

In this paper, a new discrete reaching law with a disturbance compensator has been presented. A discrete sliding-mode controller is designed by using the proposed reaching law. The time steps for system trajectories to converge to the switching manifold are found, and the QSMD of the closed-loop system is obtained. Furthermore, the system trajectories of the closed-

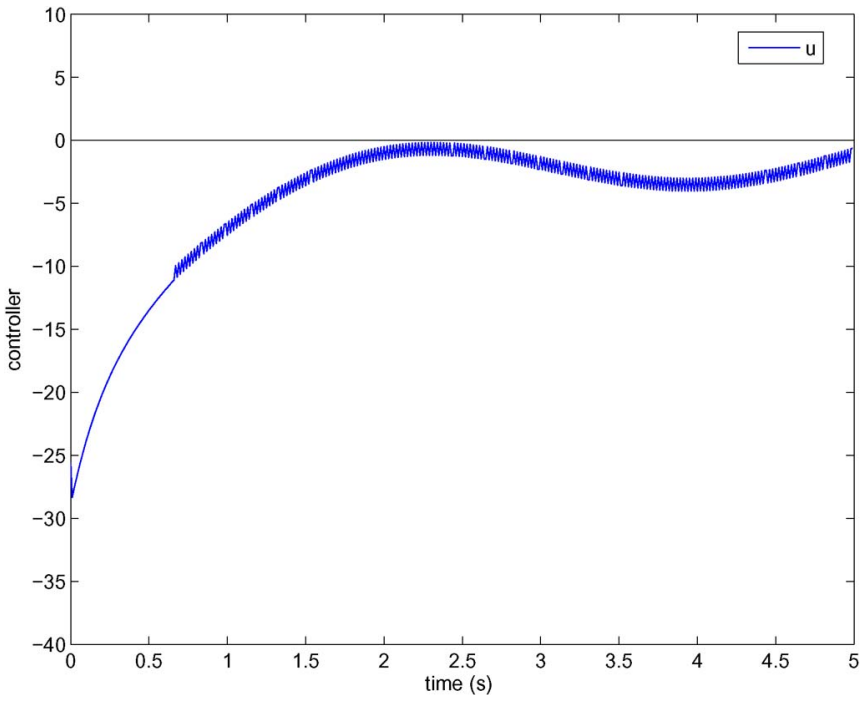

Fig. 8. Controller with the improved reaching law in the case of the sampling period $T=0.01$ in case II.

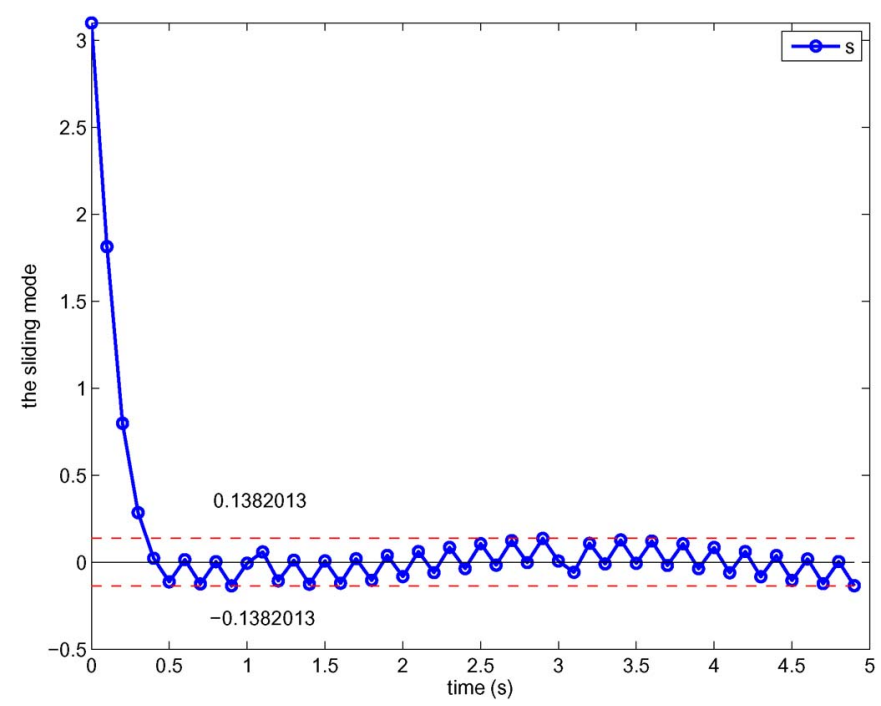

Fig. 9. Switching function with the improved reaching law in case III.

loop system in QSMD are described. Future work will focus on the nonlinear discrete systems with DSMC and industry applications.

\section{ACKNOWLEDGMENT}

The authors would like to thank the anonymous reviewers and the Associate Editor for their many helpful comments.

\section{REFERENCES}

[1] B. Draženović, "The invariance conditions in variable structure systems," Automatica, vol. 5, no. 3, pp. 287-295, May 1969.

[2] C. Milosavljevic, "General conditions of the existence of a quasi-sliding mode on the switching hyperplane in discrete variable structure systems," Autom. Remote Control, vol. 46, no. 3, pp. 307-314, 1985.

[3] V. I. Utkin, Sliding Modes in Control and Optimization. Berlin, Germany: Springer-Verlag, 1992.

[4] C. Edwards and S. Spurgeon, Sliding Mode Control: Theory and Applications. London, U.K.: Taylor \& Francis, 1998. 
[5] O. Kaynak and A. Denker, "Discrete-time sliding mode control in the presence of system uncertainty," Int. J. Control, vol. 57, no. 5, pp. 11771189, May 1993.

[6] S. Z. Sarpturk, Y. Istefanopulos, and O. Kaynak, "On the stability of discrete time sliding mode control systems," IEEE Trans. Autom. Control, vol. 32, no. 10, pp. 930-932, Oct. 1987.

[7] K. Furuta, "Sliding mode control of a discrete system," Syst. Control Lett., vol. 14, no. 2, pp. 145-152, Feb. 1990.

[8] W. Gao and J. C. Hung, "Variable structure control of nonlinear systems: A new approach," IEEE Trans. Ind. Electron., vol. 40, no. 1, pp. 45-55, Feb. 1993.

[9] W. Gao, Y. Wang, and A. Homaifa, "Discrete-time variable structure control systems," IEEE Trans. Ind. Electron., vol. 42, no. 2, pp. 117-122, Apr. 1995.

[10] A. J. Bartoszewicz, "Remark on "Discrete-time variable structure control systems"," IEEE Trans. Ind. Electron., vol. 43, no. 1, pp. 235-238, Jan. 1996.

[11] A. J. Bartoszewicz, "Discrete time quasi-sliding mode control strategies," IEEE Trans. Ind. Electron., vol. 45, no. 4, pp. 633-637, Apr. 1998.

[12] M. C. Saaj, B. Bandyopashyay, and H. Unbehauen, "A new algorithm for discrete-time sliding-mode control using fast output sampling feedback," IEEE Trans. Ind. Electron., vol. 49, no. 3, pp. 518-523, Jun. 2002.

[13] G. Bartolini, A. Ferrara, and V. I. Utkin, "Adaptive sliding mode control in discrete-time systems," Automatica, vol. 31, no. 5, pp. 769-773, May 1995.

[14] B. Veselić, B. Perunićić-Draženović, and Č. Milosavljević, "Improved discrete-time sliding-mode position control using Euler velocity estimation," IEEE Trans. Ind. Electron., vol. 57, no. 11, pp. 3840-3847, Nov. 2010

[15] W. C. Su, S. V. Drakunov, and Ü. Özgüner, "An $\mathrm{O}\left(\mathrm{T}_{2}\right)$ boundary layer in sliding mode for sampled-data systems," IEEE Trans. Autom. Control, vol. 45, no. 3, pp. 482-485, Mar. 2000.

[16] X. Yu and G. Chen, "Discretization behaviors of equivalent control based sliding-mode control systems," IEEE Trans. Autom. Control, vol. 48, no. 9, pp. 1641-1646, Sep. 2003.

[17] Z. Galias and X. Yu, "Euler's discretization of single input sliding-mode control systems," IEEE Trans. Autom. Control, vol. 52, no. 9, pp. 17261730, Sep. 2007.

[18] X. Xia and A. S. I. Zinober, " $\Delta$-modulated feedback in discretization of sliding mode control," Automatica, vol. 42, no. 5, pp. 771-776, May 2006.

[19] X. Xia and A. S. I. Zinober, "Periodic orbits from $\Delta$-modulation of stable linear systems," IEEE Trans. Autom. Control, vol. 49, no. 8, pp. 13761380, Aug. 2004.

[20] X. Yu, B. Wang, Z. Galias, and G. Chen, "Discretization effect on equivalent control based multi-input sliding mode control systems," IEEE Trans. Autom. Control, vol. 53, no. 6, pp. 1563-1569, Jul. 2008.

[21] B. Wang, X. Yu, and X. Li, "ZOH discretization effect on higher-order sliding mode control systems," IEEE Trans. Ind. Electron., vol. 55, no. 11, pp. 4055-4064, Nov. 2008.

[22] O. Kaynak, A. Bartoszewicz, and V. I. Utkin, "Guest editorial: Industrial applications of sliding mode control-Part II," IEEE Trans. Ind. Electron., vol. 56, no. 9, pp. 3271-3274, Sep. 2009.

[23] V. Acary and B. Brogliato, "Implicit Euler numerical scheme and chattering-free implementation of sliding mode systems," Syst. Control Lett., vol. 59, pp. 284-293, Sep. 2010.

[24] V. Acary, B. Brogliato, and Y. Orlov, "Chattering-free digital sliding-mode control with state observer and disturbance rejection," IEEE Trans. Autom Control, vol. 57, no. 5, pp. 1087-1101, May 2012.

[25] H. Oza, V. Acary, Y. Orlov, S. Spurgeon, and B. Brogliato, "Finite time tracking of unilaterally constrained planar systems with pre-specified settling time: Second order sliding mode synthesis and chattering-free digital implementation," in Proc. IEEE Conf. Decision Control, Maui, HI, USA, Dec. 10-13, 2012, pp. 5471-5476.

[26] R. Morgan and Ü. Özgüner, "A decentralized variable structure control algorithm for robotic manipulators," IEEE Trans. Robot. Autom., vol. RA-1, no. 1, pp. 57-65, Mar. 1985.

[27] K. Youcef-Toumi and O. Itô, "A time delay controller systems with unknown dynamics," J. Dyn. Syst. Meas. Control, vol. 112, no. 1, pp. $133-$ 142, Mar. 1990.

[28] X. Xia and G. Chen, "On delta-modulated control: A simple system with complex dynamics," Chaos Solitons Fractals, vol. 33, no. 4, pp. 13141328, Aug. 2007.

[29] J. Yang and X. Yu, "Sliding-mode control for systems with mismatched uncertainties via a disturbance observer," IEEE Trans. Ind. Electron., vol. 60, no. 1, pp. 160-169, Jan. 2013.
[30] R. J. Lian, "Enhanced adaptive self-organizing fuzzy sliding-mode controller for active suspension systems," IEEE Trans. Ind. Electron., vol. 60, no. 3, pp. 958-968, Mar. 2013.

[31] L. M. Capisani, "Trajectory planning and second-order sliding mode motion/interaction control for robot manipulators in unknown environments," IEEE Trans. Ind. Electron., vol. 59, no. 8, pp. 3189-3198, Aug. 2012.

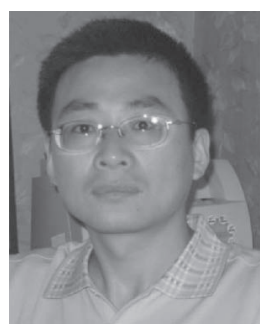

Shaocheng Qu (M'13) received the B.Sc. degree in process control and instrument from Wuhan University of Chemical and Technology, Wuhan, China, in 1994, the M.Sc. degree in control engineering from the Naval University of Engineering, Wuhan, in 2000 , and the Ph.D. degree in control theory and control engineering from Huazhong University of Science and Technology, Wuhan, in 2004.

$\mathrm{He}$ is currently a Full Professor with the Department of Electronics and Information Engineering, College of Physical Science and Technology, Central China Normal University, Wuhan. His main research interests are in the areas of sliding-mode-control (SMC) theory and applications, system control, and embedded systems. He has published over 50 papers on these topics and provided consultancy to various industries. He has also published a monograph on SMC theory and applications (in Chinese).

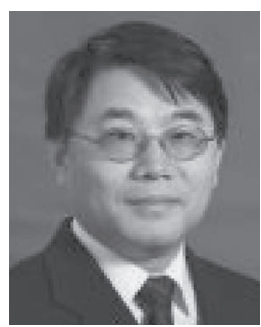

Xiaohua Xia (M'98-SM'98-F'08) received the B.Sc. degree in applied mathematics from Wuhan Institute of Hydraulic and Electrical Engineering, Wuhan, China, in 1983 and the Ph.D. degree in automatic control theory and application from Beijing University of Aeronautics and Astronautics, Beijing, China, in 1989.

$\mathrm{He}$ is currently a Professor with the Department of Electrical, Electronics and Computer Engineering, University of Pretoria, Pretoria, South Africa, where he is the Director of the Centre of New Energy Systems and the National Hub for the Postgraduate Program in Energy Efficiency and Demand-side Management. Before joining the University of Pretoria in 1998, he was academically affiliated with the University of Stuttgart, Stuttgart, Germany, the Ecole Centrale de Nantes, Nantes, France, and the National University of Singapore, Singapore. He has been an Associate Editor of Automatica and a Specialist Editor (control) of the South African Institute of Electrical Engineers Africa Research Journal.

Prof. Xia is a fellow of the South African Academy of Engineering, and he has an A rating from the South African National Research Foundation. He also serves as the Chair of the Technical Committee of Nonlinear Systems of the International Federation of Automatic Control. He has been an Associate Editor of the IEEE TRANSACTIONS ON CIRCUITS AND SYSTEMS II and the IEEE TRANSACTIONS ON AUTOMatic CONTROL.

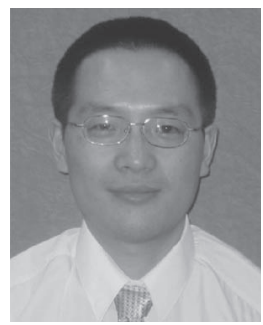

Jiangfeng Zhang received the B.Sc. and Ph.D. degrees in computing mathematics from Xian Jiaotong University, Xi'an, China, in 1995 and 1999, respectively

From 2000 to 2002, he was a Lecturer with Shanghai Jiaotong University, Shanghai, China. Then, he was a Postdoctoral Researcher with The Chinese University of Hong Kong, Shatin, Hong Kong, the Ecole Centrale de Nantes, Nantes, France, Nanyang Technological University, Singapore, and the University of Liverpool, Liverpool, U.K. After spending seven years at the University of Pretoria Republic of South Africa (RSA), Pretoria, South Africa, as a Research Fellow, a Senior Lecturer, and an Associate Professor, he joined the University of Strathclyde, Glasgow, U.K., in late 2013 as a Senior Lecturer. His current research interests are energy management and control theory.

Dr. Zhang is a certified energy manager, and a certified measurement and verification professional. 